\title{
MINORS OF A SKEW SYMMETRIC MATRIX: A COMBINATORIAL APPROACH*
}

\author{
LARS WINTHER CHRISTENSEN ${ }^{\dagger}$, OANA VELICHE $^{\ddagger}$, AND JERZY WEYMAN ${ }^{\S}$
}

\begin{abstract}
Knuth's combinatorial approach to Pfaffians is used to reprove and clarify a century-old formula, due to Brill. It expresses arbitrary minors of a skew symmetric matrix in terms of Pfaffians.
\end{abstract}

Key words. Pfaffian, Minor.

AMS subject classifications. 15A15, 15A24.

1. Introduction. In a paper [4] from 1996, Knuth took a combinatorial approach to Pfaffians. It was immediately noticed that this approach facilitates generalizations and simplified proofs of several known identities involving Pfaffians; see for example Hamel [3].

In this short note, we apply the same approach to reprove a formula that expresses arbitrary minors of a skew symmetric matrix in terms of Pfaffians. The formula, which we here state as Theorem 2.1, first appeared in a 1904 paper by Brill [1]. Our goal here is not merely to give a short modern proof but also to clarify two aspects of the formula: (1) To compute minors with Brill's formula [1, equation (1)] one needs to apply a sign convention explained in a footnote; we integrate this sign into the formula. (2) As Brill states right at the top of his paper, one can reduce to the case of minors with disjoint row and column sets, and his formula deals only with that case. We build that reduction into our proof and arrive at a formula that also holds for minors with overlapping row and column sets.

Bibliometric data suggests that Brill's formula may have gone largely unnoticed. For us it turned out to be key to clarify a computation in commutative algebra [2].

2. Brill's formula. Let $T=\left(t_{i j}\right)$ be an $n \times n$ skew symmetric matrix with entries in some commutative ring. Assume that $T$ has zeros on the diagonal; this is, of course, automatic if the characteristic of the ring is not 2. Set $\mathcal{P}[i j]=t_{i j}$ for $i, j \in\{1, \ldots, n\}$ and extend $\mathcal{P}$ to a function on words in letters from the set $\{1, \ldots, n\}$ as follows:

$$
\mathcal{P}\left[i_{1} \cdots i_{m}\right]= \begin{cases}0 & \text { if } m \text { is odd } \\
\sum \operatorname{sgn}\left(\begin{array}{c}
i_{1} \cdots i_{2 k} \\
j_{1} \cdots j_{2 k}
\end{array}\right) \mathcal{P}\left[j_{1} j_{2}\right] \cdots \mathcal{P}\left[j_{2 k-1} j_{2 k}\right] & \text { if } m=2 k \text { is even }\end{cases}
$$

where the sum is over all partitions of $\left\{i_{1}, \ldots, i_{2 k}\right\}$ into $k$ subsets of cardinality 2 . The order of the two elements in each subset is irrelevant as the difference in sign $\mathcal{P}\left[j j^{\prime}\right]=-\mathcal{P}\left[j^{\prime} j\right]$ is offset by a change of sign of

\footnotetext{
* Received by the editors on May 25, 2020. Accepted for publication on July 28, 2020. Handling Editor: João Filipe Queiró. Corresponding Author: Lars Winther Christensen.

$\dagger$ Texas Tech University, Lubbock, TX 79409, USA (lars.w.christensen@ttu.edu, http://www.math.ttu.edu/ lchriste). Partially supported by Simons Foundation collaboration grant no. 428308.

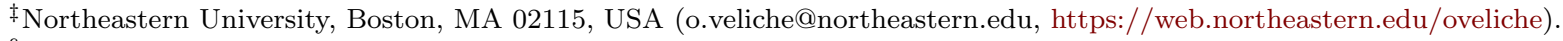

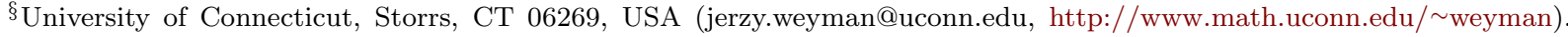
Partially supported by NSF DMS grant no. 1802067.
} 
Electronic Journal of Linear Algebra, ISSN 1081-3810

A publication of the International Linear Algebra Society

Volume 36, pp. 658-663, September 2020.

the permutation; see [4, Section 0$]$. The value of $\mathcal{P}$ on the empty word is by convention 1 , and the value of $\mathcal{P}$ on a word with a repeated letter is 0 . The latter is a convention in characteristic 2 and otherwise automatic.

For subsets $R$ and $S$ of $\{1, \ldots, n\}$ we write $T[R ; S]$ for the submatrix of $T$ obtained by taking the rows indexed by $R$ and the columns indexed by $S$. The function $\mathcal{P}$ computes the Pfaffians of skew symmetric submatrices of $T$. Indeed, for a subset $R \subseteq\{1, \ldots, n\}$ with elements $r_{1}<\cdots<r_{m}$ one has

$$
\operatorname{Pf}(T[R ; R])=\mathcal{P}\left[r_{1} \cdots r_{m}\right]
$$

With this approach to Pfaffians, Knuth [4] gives elegant proofs for several classic formulas, generalizing them and clarifying sign conventions in the process through the introduction of permutation signs. For a word $\alpha$ in letters from $\{1, \ldots, n\}$ and $a \in \alpha$ the following variation on the classic Laplacian expansion is obtained as a special case of a formula ascribed to Tanner [5],

$$
\mathcal{P}[\alpha]=\sum_{x \in \alpha} \operatorname{sgn}(\underset{a x(\alpha \backslash a x)}{\alpha}) \mathcal{P}[a x] \mathcal{P}[\alpha \backslash a x] ;
$$

see [4, equation (2.0)]. The introduction of the permutation sign is what facilitates our statement and proof of Brill's formula.

THEOREM 2.1. Let $T$ be an $n \times n$ skew symmetric matrix. Let $R$ and $S$ be subsets of the set $\{1, \ldots, n\}$ with elements $r_{1}<\cdots<r_{m}$ and $s_{1}<\cdots<s_{m}$. With

$$
\rho=r_{1} \cdots r_{m} \quad \text { and } \quad \sigma=s_{1} \cdots s_{m}
$$

the next equality holds:

$$
\operatorname{det}(T[R ; S])=(-1)^{\left\lfloor\frac{m}{2}\right\rfloor} \sum_{k=0}^{\left\lfloor\frac{m}{2}\right\rfloor}(-1)^{k} \sum_{\substack{|\omega|=2 k \\
\omega \subseteq \rho}} \operatorname{sgn}\left(\begin{array}{c}
\rho \\
\omega(\rho \backslash \omega)
\end{array}\right) \mathcal{P}[\omega] \mathcal{P}[(\rho \backslash \omega) \sigma]
$$

Notice that only subwords $\omega$ of $\rho$ that contain $\rho \cap \sigma$ contribute to the sum, otherwise the word $(\rho \backslash \omega) \sigma$ has a repeated letter. The quantity $\mathcal{P}[\omega]$ is the Pfaffian of the submatrix $\operatorname{Pf}(T[\{\omega\} ;\{\omega\}])$, where $\{\omega\}$ is the subset of $\{1, \ldots, n\}$ whose elements are the letters in $\omega$. Similarly, for a word $\omega$ that contains $\rho \cap \sigma$, the quantity $\mathcal{P}[(\rho \backslash \omega) \sigma]$ equals sgn $(\underset{(\alpha \backslash \sigma) \sigma}{\alpha}) \operatorname{Pf}(T[\{\alpha\} ;\{\alpha\}])$ where the word $\alpha$ has the letters from $(\rho \backslash \omega) \sigma$ written in increasing order.

3. Our proof. For subsets $R$ and $S$ as in the statement set

$$
\mathcal{D}(\rho ; \sigma)=\operatorname{det}(T[R ; S])
$$

For a word $\alpha$ in letters from $\{1, \ldots, n\}$ we write $\bar{\alpha}$ for the word that has the letters from $\alpha$ written in increasing order. In the words $\rho$ and $\sigma$ and their subwords the letters already appear in increasing order, but the bar notation comes in handy for concatenated words such as $\rho \sigma$.

3.1. Disjoint row and column sets. We first assume that $\rho$ and $\sigma$ are disjoint subwords of $1 \cdots n$. Under this assumption $\mathcal{P}[(\rho \backslash \omega) \sigma]=\mathcal{P}[\rho \sigma \backslash \omega]$ holds for every subword $\omega \subseteq \rho$, and in the balance of the 
section we use the simpler notation $\mathcal{P}[\rho \sigma \backslash \omega]$. We proceed by induction on $m$. For $m=1$ the formula holds as one has $\operatorname{det}\left(T\left[\left\{r_{1}\right\} ;\left\{s_{1}\right\}\right]\right)=t_{r_{1} s_{1}}=\mathcal{P}\left[r_{1} s_{1}\right]$. For $m=2$ expansion of the determinant along row $r_{1}$ yields

$$
\mathcal{D}(\rho ; \sigma)=\mathcal{P}\left[r_{1} s_{1}\right] \mathcal{P}\left[r_{2} s_{2}\right]-\mathcal{P}\left[r_{1} s_{2}\right] \mathcal{P}\left[r_{2} s_{1}\right]
$$

With $\alpha=\rho \sigma$ and $a=r_{1}$ the formula (2.1) reads

$$
\begin{aligned}
\mathcal{P}[\rho \sigma] & =\sum_{x \in \rho \sigma} \operatorname{sgn}\left(\begin{array}{c}
\rho \sigma \\
r_{1} x\left(\rho \sigma \backslash r_{1} x\right)
\end{array}\right) \mathcal{P}\left[r_{1} x\right] \mathcal{P}\left[\rho \sigma \backslash r_{1} x\right] \\
& =\mathcal{P}\left[r_{1} r_{2}\right] \mathcal{P}\left[s_{1} s_{2}\right]-\mathcal{P}\left[r_{1} s_{1}\right] \mathcal{P}\left[r_{2} s_{2}\right]+\mathcal{P}\left[r_{1} s_{2}\right] \mathcal{P}\left[r_{2} s_{1}\right] \\
& =\mathcal{P}[\rho] \mathcal{P}[\sigma]-\mathcal{D}(\rho ; \sigma),
\end{aligned}
$$

which can be rewritten in the desired form:

$$
\mathcal{D}(\rho ; \sigma)=-(\mathcal{P}[\rho \sigma]-\mathcal{P}[\rho] \mathcal{P}[\sigma])
$$

Before we move on to the induction step, we record another consequence of (2.1):

$$
\begin{aligned}
\mathcal{P}[\rho \sigma \backslash \omega]= & \sum_{r \in \rho \backslash r_{1} \omega} \operatorname{sgn}\left(\begin{array}{c}
\rho \sigma \backslash \omega \\
r_{1} r\left(\rho \sigma \backslash \omega r_{1} r\right)
\end{array}\right) \mathcal{P}\left[r_{1} r\right] \mathcal{P}\left[\rho \sigma \backslash \omega r_{1} r\right] \\
& +\sum_{s \in \sigma} \operatorname{sgn}\left(\begin{array}{c}
\rho \sigma \backslash \omega \\
r_{1} s\left(\rho \sigma \backslash \omega r_{1} s\right)
\end{array}\right) \\
= & \sum_{r \in \rho \backslash r_{1} \omega} \operatorname{sgn}\left(\begin{array}{c}
\rho \backslash \omega \\
r_{1} r\left(\rho \backslash \omega r_{1} r\right)
\end{array}\right) \mathcal{P}\left[r_{1} r\right] \mathcal{P}\left[\rho \sigma \backslash \omega r_{1} s\right] \\
& +(-1)^{|\rho \backslash \omega|-1} \sum_{s \in \sigma} \operatorname{sgn}\left(\begin{array}{c}
\sigma \\
s(\sigma \backslash s)
\end{array}\right) \mathcal{P}\left[r_{1} s\right] \mathcal{P}\left[\rho \sigma \backslash \omega r_{1} r\right]
\end{aligned}
$$

Now let $m \geq 2$ and $|\rho|=m+1=|\sigma|$. In the next computation, the first equality is the expansion of the determinant $\mathcal{D}(\rho ; \sigma)$ along row $r_{1}$. The second equality follows from the induction hypothesis, and the third is obtained by changing the order of summation. The fourth equality follows from (3.2). The fifth equality uses that $|\omega|$ is even and that $|\rho \backslash \omega|-1=m-2 k \equiv m \bmod 2$ holds.

$$
\begin{aligned}
\mathcal{D}(\rho ; \sigma)= & \sum_{s \in \sigma} \operatorname{sgn}\left(\begin{array}{c}
\sigma \\
s \sigma \backslash s
\end{array}\right) \mathcal{P}\left[r_{1} s\right] \mathcal{D}\left(\rho \backslash r_{1} ; \sigma \backslash s\right) \\
= & \sum_{s \in \sigma} \operatorname{sgn}\left(\begin{array}{c}
\sigma \\
s \sigma \backslash s
\end{array}\right) \mathcal{P}\left[r_{1} s\right] \\
& \cdot\left((-1)\left\lfloor\frac{m}{2}\right\rfloor \sum_{k=0}^{\left\lfloor\frac{m}{2}\right\rfloor}(-1)^{k} \sum_{\substack{|\omega|=2 k \\
\omega \subseteq \rho \backslash r_{1}}} \operatorname{sgn}\left(\begin{array}{c}
\rho \backslash r_{1} \\
\omega\left(\rho \backslash r_{1} \omega\right)
\end{array}\right) \mathcal{P}[\omega] \mathcal{P}\left[\rho \sigma \backslash r_{1} \omega s\right]\right) \\
= & (-1)^{\left\lfloor\frac{m}{2}\right\rfloor} \sum_{k=0}^{\left\lfloor\frac{m}{2}\right\rfloor}(-1)^{k} \sum_{\substack{\mid \omega\rfloor=2 k \\
\omega \subseteq \rho \backslash r_{1}}} \operatorname{sgn}\left(\begin{array}{c}
\rho \backslash r_{1} \\
\omega\left(\rho \backslash r_{1} \omega\right)
\end{array}\right) \mathcal{P}[\omega] \\
& \cdot\left(\sum_{s \in \sigma} \operatorname{sgn}\left(\begin{array}{c}
\sigma \\
s \sigma \backslash s
\end{array}\right) \mathcal{P}\left[r_{1} s\right] \mathcal{P}\left[\rho \sigma \backslash r_{1} \omega s\right]\right)
\end{aligned}
$$


Electronic Journal of Linear Algebra, ISSN 1081-3810

A publication of the International Linear Algebra Society

Volume 36, pp. 658-663, September 2020.

$$
\begin{aligned}
& =(-1)^{\left\lfloor\frac{m}{2}\right\rfloor} \sum_{k=0}^{\left\lfloor\frac{m}{2}\right\rfloor}(-1)^{k} \sum_{\substack{|\omega|=2 k \\
\omega \subseteq \rho \backslash r_{1}}} \operatorname{sgn}\left(\begin{array}{c}
\rho \backslash r_{1} \\
\omega\left(\rho \backslash r_{1} \omega\right)
\end{array}\right) \mathcal{P}[\omega] \\
& \cdot(-1)^{|\rho \backslash \omega|-1}\left(\mathcal{P}[\rho \sigma \backslash \omega]-\sum_{r \in \rho \backslash \omega r_{1}} \operatorname{sgn}\left(\begin{array}{c}
\rho \backslash \omega \\
r_{1} r\left(\rho \backslash \omega r_{1} r\right)
\end{array}\right) \mathcal{P}\left[r_{1} r\right] \mathcal{P}\left[\rho \sigma \backslash \omega r_{1} r\right]\right) \\
& =(-1)^{\left\lfloor\frac{m}{2}\right\rfloor+m}\left(\sum_{k=0}^{\left\lfloor\frac{m}{2}\right\rfloor}(-1)^{k} \sum_{\substack{|\omega|=2 k \\
\omega \subseteq \rho \backslash r_{1}}} \operatorname{sgn}\left(\begin{array}{c}
\rho \\
\omega(\rho \backslash \omega)
\end{array}\right) \mathcal{P}[\omega] \mathcal{P}[\rho \sigma \backslash \omega]-\sum_{k=0}^{\left\lfloor\frac{m}{2}\right\rfloor}(-1)^{k}\right. \\
& \left.\cdot \sum_{\substack{|\omega|=2 k \\
\omega \subseteq \rho \backslash r_{1}}} \operatorname{sgn}\left(\begin{array}{c}
\rho \\
\omega(\rho \backslash \omega)
\end{array}\right) \mathcal{P}[\omega] \sum_{r \in \rho \backslash \omega r_{1}} \operatorname{sgn}\left(\begin{array}{c}
\rho \backslash \omega \\
r_{1} r\left(\rho \backslash \omega r_{1} r\right)
\end{array}\right) \mathcal{P}\left[r_{1} r\right] \mathcal{P}\left[\rho \sigma \backslash \omega r_{1} r\right]\right) \\
& =(-1)^{\left\lfloor\frac{m+1}{2}\right\rfloor}\left(\sum_{k=0}^{\left\lfloor\frac{m}{2}\right\rfloor}(-1)^{k} \sum_{\substack{|\omega|=2 k \\
\omega \subseteq \rho \backslash r_{1}}} \operatorname{sgn}\left(\begin{array}{c}
\rho \\
\omega(\rho \backslash \omega)
\end{array}\right) \mathcal{P}[\omega] \mathcal{P}[\rho \sigma \backslash \omega]+\sum_{k=0}^{\left\lfloor\frac{m}{2}\right\rfloor}(-1)^{k+1}\right. \\
& \left.\cdot \sum_{\substack{|\omega|=2 k \\
\omega \subseteq \rho \backslash r_{1}}} \sum_{r \in \rho \backslash \omega r_{1}} \operatorname{sgn}\left(\underset{\omega r_{1} r\left(\rho \backslash \omega r_{1} r\right)}{\rho}\right) \mathcal{P}[\omega] \mathcal{P}\left[r_{1} r\right] \mathcal{P}\left[\rho \sigma \backslash \omega r_{1} r\right]\right) .
\end{aligned}
$$

The next step is to simplify the last line in the display above. The first equality in the following computation holds as $|\omega|$ is even. The third equality follows by substituting $\omega^{\prime}$ for the word $\overline{r_{1} r \omega}$ and noticing that one then has

$$
\operatorname{sgn}\left(\begin{array}{c}
\rho \\
r_{1} r \omega\left(\rho \backslash r_{1} r \omega\right)
\end{array}\right)=\operatorname{sgn}\left(\begin{array}{c}
\rho \\
\omega^{\prime}\left(\rho \backslash \omega^{\prime}\right)
\end{array}\right) \operatorname{sgn}\left(\begin{array}{c}
\omega^{\prime} \\
r_{1} r \omega
\end{array}\right)=\operatorname{sgn}\left(\begin{array}{c}
\rho \\
\omega^{\prime}\left(\rho \backslash \omega^{\prime}\right)
\end{array}\right) \operatorname{sgn}\left(\begin{array}{c}
\omega^{\prime} \\
r_{1} r\left(\omega^{\prime} \backslash r_{1} r\right)
\end{array}\right) .
$$

The last equality follows from (2.1) applied with $\alpha=\omega^{\prime}$ and $a=r_{1}$.

$$
\begin{aligned}
& \sum_{\substack{|\omega|=2 k \\
\omega \subseteq \rho \backslash r_{1}}} \sum_{r \in \rho \backslash \omega r_{1}} \operatorname{sgn}\left(\underset{\omega r_{1} r\left(\rho \backslash \omega r_{1} r\right)}{\rho}\right) \mathcal{P}[\omega] \mathcal{P}\left[r_{1} r\right] \mathcal{P}\left[\rho \sigma \backslash \omega r_{1} r\right] \\
& =\sum_{\substack{|\omega|=2 k \\
\omega \subseteq \rho \backslash r_{1}}} \sum_{r \in \rho \backslash \omega r_{1}} \operatorname{sgn}\left({ }_{r_{1} r \omega\left(\rho \backslash r_{1} r \omega\right)}^{\rho}\right) \mathcal{P}[\omega] \mathcal{P}\left[r_{1} r\right] \mathcal{P}\left[\rho \sigma \backslash r_{1} r \omega\right] \\
& =\sum_{\substack{\left|\omega^{\prime}\right|=2 k+2 \\
r_{1} \in \omega^{\prime} \subseteq \rho}} \sum_{r \in \omega^{\prime} \backslash r_{1}} \operatorname{sgn}\left(\begin{array}{c}
\rho \\
\omega^{\prime}\left(\rho \backslash \omega^{\prime}\right)
\end{array}\right) \operatorname{sgn}\left(\begin{array}{c}
\omega_{r_{1}} r\left(\omega^{\prime} \backslash r_{1} r\right)
\end{array}\right) \mathcal{P}\left[\omega^{\prime} \backslash r_{1} r\right] \mathcal{P}\left[r_{1} r\right] \mathcal{P}\left[\rho \sigma \backslash \omega^{\prime}\right] \\
& =\sum_{\substack{\left|\omega^{\prime}\right|=2 k+2 \\
r_{1} \in \omega^{\prime} \subseteq \rho}} \operatorname{sgn}\left(\begin{array}{c}
\rho \\
\omega^{\prime}\left(\rho \backslash \omega^{\prime}\right)
\end{array}\right) \mathcal{P}\left[\rho \sigma \backslash \omega^{\prime}\right]\left(\sum_{r \in \omega^{\prime} \backslash r_{1}} \operatorname{sgn}\left(\underset{r_{1} r\left(\omega^{\prime} \backslash r_{1} r\right)}{\omega^{\prime}}\right) \mathcal{P}\left[r_{1} r\right] \mathcal{P}\left[\omega^{\prime} \backslash r_{1} r\right]\right) \\
& =\sum_{\substack{\left|\omega^{\prime}\right|=2(k+1) \\
r_{1} \in \omega^{\prime} \subseteq \rho}} \operatorname{sgn}\left(\begin{array}{c}
\rho \\
\omega^{\prime}\left(\rho \sigma \backslash \omega^{\prime}\right)
\end{array}\right) \mathcal{P}\left[\rho \backslash \omega^{\prime}\right] \mathcal{P}\left[\omega^{\prime}\right] .
\end{aligned}
$$


Substituting this into the computation above one gets the desired equality.

$$
\begin{aligned}
\mathcal{D}(\rho ; \sigma)= & (-1)^{\left\lfloor\frac{m+1}{2}\right\rfloor}\left(\sum_{k=0}^{\left\lfloor\frac{m}{2}\right\rfloor}(-1)^{k} \sum_{\substack{|\omega|=2 k \\
\omega \subseteq \rho>r_{1}}} \operatorname{sgn}\left(\begin{array}{c}
\rho \\
\omega(\rho \backslash \omega)
\end{array}\right) \mathcal{P}[\omega] \mathcal{P}[\rho \sigma \backslash \omega]\right. \\
& \left.+\sum_{k=0}^{\left\lfloor\frac{m}{2}\right\rfloor}(-1)^{k+1} \sum_{\substack{\left|\omega^{\prime}\right|=2(k+1) \\
r_{1} \in \omega^{\prime} \subseteq \rho}} \operatorname{sgn}\left(\begin{array}{c}
\rho \\
\omega^{\prime}\left(\rho \backslash \omega^{\prime}\right)
\end{array}\right) \mathcal{P}\left[\rho \sigma \backslash \omega^{\prime}\right] \mathcal{P}\left[\omega^{\prime}\right]\right) \\
= & (-1)^{\left\lfloor\frac{m+1}{2}\right\rfloor} \sum_{k=0}^{\left\lfloor\frac{m+1}{2}\right\rfloor}(-1)^{k} \sum_{\substack{|\omega|=2 k \\
\omega \subseteq \rho}} \operatorname{sgn}\left(\begin{array}{c}
\rho \\
\omega(\rho \backslash \omega)
\end{array}\right) \mathcal{P}[\omega] \mathcal{P}[\rho \sigma \backslash \omega] .
\end{aligned}
$$

This proves the asserted formula in the special case where $R$ and $S$ are disjoint, and it remains to reduce the general case to this one.

3.2. Overlapping row and column sets. First notice that the submatrix $T[R ; S]$ agrees with a submatrix of the $2 n \times 2 n$ skew symmetric matrix $T^{\prime}$ obtained from $T$ by repeating all entries, horizontally and vertically. For example, with

$$
T=\left(\begin{array}{ccc}
0 & t_{12} & t_{13} \\
-t_{12} & 0 & t_{23} \\
-t_{13} & -t_{23} & 0
\end{array}\right) \quad \text { and } \quad T^{\prime}=\left(\begin{array}{cccccc}
0 & 0 & t_{12} & t_{12} & t_{13} & t_{13} \\
0 & 0 & t_{12} & t_{12} & t_{13} & t_{13} \\
-t_{12} & -t_{12} & 0 & 0 & t_{23} & t_{23} \\
-t_{12} & -t_{12} & 0 & 0 & t_{23} & t_{23} \\
-t_{13} & -t_{13} & -t_{23} & -t_{23} & 0 & 0 \\
-t_{13} & -t_{13} & -t_{23} & -t_{23} & 0 & 0
\end{array}\right)
$$

one has $T[\{1,2\} ;\{2,3\}]=T^{\prime}[\{1,3\} ;\{4,6\}]$. In general, one has

$$
T[R ; S]=T^{\prime}\left[R^{\prime} ; S^{\prime}\right]
$$

for

$$
R^{\prime}=\left\{2 r_{1}-1, \ldots, 2 r_{m}-1\right\} \quad \text { and } \quad S^{\prime}=\left\{2 s_{1}, \ldots, 2 s_{m}\right\}
$$

One now has

$$
\begin{aligned}
\operatorname{det}(T[R ; S]) & =\operatorname{det}\left(T^{\prime}\left[R^{\prime} ; S^{\prime}\right]\right) \\
& =(-1)^{\left\lfloor\frac{m}{2}\right\rfloor} \sum_{k=0}^{\left\lfloor\frac{m}{2}\right\rfloor}(-1)^{k} \sum_{\substack{\left|\omega^{\prime}\right|=2 k \\
\omega^{\prime} \subseteq \rho^{\prime}}} \operatorname{sgn}\left(\begin{array}{c}
\rho^{\prime} \\
\omega^{\prime}\left(\rho^{\prime} \backslash \omega^{\prime}\right)
\end{array}\right) \mathcal{P}\left[\omega^{\prime}\right] \mathcal{P}\left[\rho^{\prime} \sigma^{\prime} \backslash \omega^{\prime}\right],
\end{aligned}
$$

where the second equality holds by the case already established. Indeed, the numbers in $R^{\prime}$ are odd and the numbers in $S^{\prime}$ are even. Notice that for every $k$ there is a one-to-one correspondence, given by the relation $r_{i}^{\prime}=2 r_{i}-1$, between subwords $\omega^{\prime} \subseteq \rho^{\prime}$ of length $2 k$ and subwords $\omega$ of $\rho$ of length $2 k$, and one has $\mathcal{P}\left[\omega^{\prime}\right]=\mathcal{P}[\omega]$ for corresponding subwords. Let $U$ denote the submatrix of $T^{\prime}\left[R^{\prime}, S^{\prime}\right]$ obtained by removing the rows and columns whose indices, all odd, appear in the word $\omega^{\prime}$. One has

$$
\mathcal{P}\left[\rho^{\prime} \sigma^{\prime} \backslash \omega^{\prime}\right]=\operatorname{sgn}\left(\begin{array}{c}
\overline{\rho^{\prime} \sigma^{\prime} \backslash \omega^{\prime}} \\
\rho^{\prime} \sigma^{\prime} \backslash \omega^{\prime}
\end{array}\right) \operatorname{Pf}(U) .
$$


If an element $x \in R \cap S$ does not appear in $\omega$, then $2 x-1$ is not in $\omega^{\prime}$, so $U$ contains the identical rows/columns $2 x-1$ and $2 x$ from $T^{\prime}$, whence $\mathcal{P}\left[\rho^{\prime} \sigma^{\prime} \backslash \omega^{\prime}\right]=0=\mathcal{P}[(\rho \backslash \omega) \sigma]$ holds. On the other hand, if every element of $R \cap S$ appears in $\omega$, then the matrix $U$ is a submatrix of $T[R \backslash R \cap S ; S \backslash R \cap S]$ with Pfaffian $\operatorname{sgn}\left(\begin{array}{c}\overline{(\rho \backslash \omega) \sigma} \\ (\rho \backslash \omega) \sigma\end{array}\right) \mathcal{P}[(\rho \backslash \omega) \sigma]$. As one has $\rho^{\prime} \sigma^{\prime} \backslash \omega^{\prime}=\left(\rho^{\prime} \backslash \omega^{\prime}\right) \sigma^{\prime}$ the permutations $\left(\begin{array}{c}\overline{(\rho \backslash \omega) \sigma} \\ (\rho \backslash \omega) \sigma\end{array}\right)$ and $\left(\begin{array}{c}\overline{\rho^{\prime} \sigma^{\prime} \backslash \omega^{\prime}} \\ \rho^{\prime} \sigma^{\prime} \backslash \omega^{\prime}\end{array}\right)$ have the same sign, and the proof is complete.

\section{REFERENCES}

[1] J. Brill. On the minors of a skew-symmetrical determinant. Proc. London Math. Soc., 2(1):103-111, 1904.

[2] L.W. Christensen, O. Veliche, and J. Weyman. Three takes of almost complete intersections of codimension 3. In preparation.

[3] A.M. Hamel. Pfaffian identities: A combinatorial approach. J. Combin. Theory Ser. A, 94(2):205-217, 2001.

[4] D.E. Knuth. Overlapping Pfaffians. Electron. J. Combin., 3(2), 1996.

[5] H.W. Lloyd Tanner. A theorem relating to Pfafffians. Messenger of Mathematics, 8:56-59, 1878. 